\title{
Bacterial infection remodels the DNA methylation landscape of human dendritic cells
}

\begin{abstract}
Alain Pacis, ${ }^{1,2}$ Ludovic Tailleux, ${ }^{3}$ Alexander M. Morin, ${ }^{4}$ John Lambourne, ${ }^{5}$ Julia L. Maclsaac, ${ }^{4}$ Vania Yotova, ${ }^{1}$ Anne Dumaine, ${ }^{1}$ Anne Danckaert, ${ }^{6}$ Francesca Luca, ${ }^{7}$ Jean-Christophe Grenier, ${ }^{1}$ Kasper D. Hansen, ${ }^{8}$ Brigitte Gicquel, ${ }^{3}$ Miao Yu, ${ }^{9}$ Athma Pai, ${ }^{10}$ Chuan He, ${ }^{9}$ Jenny Tung, ${ }^{11}$ Tomi Pastinen, ${ }^{5}$ Michael S. Kobor, ${ }^{4}$ Roger Pique-Regi, ${ }^{7}$ Yoav Gilad, ${ }^{12}$ and Luis B. Barreiro ${ }^{1,13}$

${ }^{1}$ Department of Genetics, CHU Sainte-Justine Research Center, Montreal, H3T1C5 Canada; ${ }^{2}$ Department of Biochemistry, University of Montreal, Montreal, H3T1/4 Canada; Institut Pasteur, Mycobacterial Genetics Unit, Paris, 75015 France; ${ }^{4}$ Centre for Molecular Medicine and Therapeutics, Child and Family Research Institute, Department of Medical Genetics, University of British Columbia, Vancouver, British Columbia, V6T1Z4, Canada; ${ }^{5}$ Génome Québec Innovation Centre, Department of Human Genetics, McGill University, Montreal, H3A0G1 Canada; ${ }^{6}$ Institut Pasteur, Imagopole, Paris, 75015 France; ${ }^{7}$ Center for Molecular Medicine and Genetics and Department of Obstetrics and Gynecology, Wayne State University, Detroit, Michigan 48202, USA; ${ }^{8}$ Department of Biostatistics and McKusick-Nathans Institute for Genetic Medicine, Johns Hopkins Bloomberg School of Public Health, Baltimore, Maryland 21205, USA; ${ }^{9}$ Department of Chemistry and Institute for Biophysical Dynamics, University of Chicago, Chicago, Illinois 60637, USA; ${ }^{10}$ Department of Biology, Massachusetts Institute of Technology, Cambridge, Massachusetts 02139, USA;

${ }^{11}$ Departments of Evolutionary Anthropology and Biology and Duke Population Research Institute, Duke University, Durham, North Carolina 27708, USA; ${ }^{12}$ Department of Human Genetics, University of Chicago, Chicago, Illinois 60637, USA; ${ }^{13}$ Department of Pediatrics, University of Montreal, Montreal, H3T1/4 Canada
\end{abstract}

\begin{abstract}
DNA methylation is an epigenetic mark thought to be robust to environmental perturbations on a short time scale. Here, we challenge that view by demonstrating that the infection of human dendritic cells (DCs) with a live pathogenic bacteria is associated with rapid and active demethylation at thousands of loci, independent of cell division. We performed an integrated analysis of data on genome-wide DNA methylation, histone mark patterns, chromatin accessibility, and gene expression, before and after infection. We found that infection-induced demethylation rarely occurs at promoter regions and instead localizes to distal enhancer elements, including those that regulate the activation of key immune transcription factors. Active demethylation is associated with extensive epigenetic remodeling, including the gain of histone activation marks and increased chromatin accessibility, and is strongly predictive of changes in the expression levels of nearby genes. Collectively, our observations show that active, rapid changes in DNA methylation in enhancers play a previously unappreciated role in regulating the transcriptional response to infection, even in nonproliferating cells.
\end{abstract}

[Supplemental material is available for this article.]

The first immune mechanisms recruited to defend against invading pathogens are those associated with innate immune cells, such as dendritic cells (DCs) or macrophages. Once they sense an intruder, these cells induce sophisticated transcriptional programs involving the regulation of thousands of genes, which are coordinated with the help of signal-dependent transcription factors, including NF- $\mathrm{kB} / \mathrm{Rel}, \mathrm{AP}-1$, and interferon regulatory factors (IRFs) (Medzhitov 2001; Smale 2010). The regulation of this program is achieved through a series of epigenetic changes, which are thought to modulate the access of transcription factors to specific DNA regulatory elements (Bierne et al. 2012).

The most well-studied epigenetic responses to immune stimuli involve the post-translational modification of histone tails at promoter and enhancer regions (Bierne et al. 2012; Monticelli and Natoli 2013). Histone acetylation has been shown to be essen-

Corresponding authors: luis.barreiro@umontreal.ca, gilad@uchicago
.edu

Article published online before print. Article, supplemental material, and publication date are at http://www.genome.org/cgi/doi/10.1101/gr.192005.115. tial for the activation of many pro-inflammatory genes (Ghisletti et al. 2010; Qiao et al. 2013), whereas increased activity of histone deacetylases is often associated with gene repression in the context of inflammation (Villagra et al. 2009). Moreover, recent studies suggest that the response of innate cells to different immune challenges can result in the appearance of histone marks associated with de novo enhancer elements (or latent enhancers) (Kaikkonen et al. 2013; Ostuni et al. 2013). These de novo enhancers have been postulated to contribute to a faster and stronger transcriptional response to a secondary stimulus (Ostuni et al. 2013).

In contrast, we still know remarkably little about the role of other epigenetic changes in controlling responses to infection. DNA methylation has been particularly understudied, as a consequence of the belief that methylation marks are highly stable

C 2015 Pacis et al. This article is distributed exclusively by Cold Spring Harbor Laboratory Press for the first six months after the full-issue publication date (see http://genome.cshlp.org/site/misc/terms.xhtml). After six months, it is available under a Creative Commons License (Attribution-NonCommercial 4.0 International), as described at http://creativecommons.org/licenses/by$\mathrm{nc} / 4.0 /$. 
and unlikely to respond to environmental perturbations on a short time scale (Bierne et al. 2012; Monticelli and Natoli 2013). Recent work, however, suggests that DNA methylation patterns can rapidly change in response to certain environmental cues (Klug et al. 2010; Guo et al. 2011; Dowen et al. 2012; Marr et al. 2014), raising the possibility that rapid changes in DNA methylation might play a role in innate immune responses. To date, no studies have comprehensively investigated the contribution of rapid, active changes in methylation (in contrast to passive changes during cell replication) to the regulatory programs induced by innate immune cells in response to an infectious agent. More broadly, the few studies in mammalian cells that demonstrate cell division-independent changes in DNA methylation have only focused on a small number of CpG sites and, surprisingly, have suggested that such changes are poorly predictive of changes in gene expression levels (Bruniquel and Schwartz 2003; Klug et al. 2010; Guo et al. 2011; Marr et al. 2014). Here, we report the first comprehensive epigenome and transcriptome analysis of monocyte-derived DCsprofessional antigen-presenting cells that play a central role in bridging innate and adaptive immunity-before and after in vitro infection with live pathogenic bacteria. All the data generated in this study are freely accessible via a custom web-based browser that enables easy querying and visualization of epigenetic profiles at any genomic region of interest (http://luis-barreirolab.org/ EpigenomeBrowser).

\section{Results}

\section{MTB infection induces active changes in DNA methylation in human DCs}

We infected monocyte-derived DCs from six healthy donors with a live virulent strain of Mycobacterium tuberculosis (MTB), the causative agent of tuberculosis (TB) in humans. Monocyte-derived DCs are ideally suited to study active changes in methylation because they are post-mitotic and not expected to proliferate in response to infection (Pickl et al. 1996; Ardeshna et al. 2000). To experimentally confirm this assumption, we performed a carboxyfluorescein diacetate succinimidyl ester (CFSE) proliferation assay. This method relies on the ability of the highly fluorescent dye carboxyfluorescein to incorporate within cells. Following each cell division, the equal distribution of these fluorescent molecules to progeny cells results in a halving of per-cell fluorescence levels. We did not detect any decrease in per-cell fluorescence at $18 \mathrm{~h}$ post-infection, which confirms that DCs do not proliferate after MTB infection (Fig. 1A). In contrast, we observed high rates of proliferation in our positive control, human monocytic THP-1 cells (Fig. 1A).

At $18 \mathrm{~h}$ after infection, we obtained paired data on single base-pair resolution DNA methylation levels (using whole-genome shotgun bisulfite sequencing, i.e., MethylC-seq) and genome-wide gene expression data (using mRNA sequencing, i.e., mRNA-seq) in noninfected and MTB-infected DCs. For MethylCseq data, we generated 8.6 billion single-end reads (mean of 648 \pm 110 SD million reads per sample) (Supplemental Table S1), resulting in an average coverage per CpG site of $\sim 9 \times$ for each sample. We detected an average of 24 million CpG sites in each sample, corresponding to over $80 \%$ of CpG sites in the human genome. Genome-wide methylation data between biological replicates were strongly correlated, attesting to the high quality of the data (mean $r$ across all samples $=0.86$ ) (Supplemental Fig. S1).

As expected for mammalian cells, most CpG sites were highly methylated throughout the genome except near transcription start sites (TSSs), CpG islands, and putative enhancer elements (Supplemental Fig. S2A,B). We found a significant negative correlation between gene expression levels and methylation levels around TSSs $\left(r=-0.39 ; P<1 \times 10^{-16}\right)$ (Supplemental Fig. S2C,D), highlighting the well-established role of proximal methylation in the stable silencing of gene expression. Principal component analysis of our data along with MethylC-seq data from 21 other purified cell types and tissues revealed that the DC methylome is closely related to that of other blood-derived cells, particularly cells that share a common myeloid progenitor with DCs, such as neutrophils (Supplemental Fig. S2E).

We next assessed the occurrence and the extent to which the response of DCs to a bacterial infection is accompanied by active changes in DNA methylation, using the BSmooth algorithm (Hansen et al. 2012). We defined MTB-induced differentially methylated regions (MTB-DMRs) as regions of three or more consecutive CpG sites exhibiting a significant difference in methylation between the two groups $(P<0.01)$ and an absolute mean methylation difference above 0.1 (Hansen et al. 2014). Using these criteria, we identified 3271 MTB-DMRs, corresponding to both hypermethylated regions (48\%) and hypomethylated regions (52\%) (Fig. 1B; Supplemental Table S2). To independently validate these changes, we generated methylation-sensitive pyrosequencing data on control versus MTB-infected DCs from five new individuals. We targeted $21 \mathrm{CpG}$ sites that were differentially methylated in the MethylC-seq analysis, distributed across four hypermethylated (11 CpG sites) and six hypomethylated MTBDMRs (10 CpG sites) (Supplemental Table S3). We were able to validate $100 \%$ of the hypomethylated CpG sites, with effect sizes similar to or greater than those identified in the original bisulfite sequencing analysis (Fig. 1B,C; Supplemental Fig. S3A). In contrast, we were not able to validate any of the hypermethylated CpG sites (Supplemental Fig. S3B), which indicates that most (if not all) active changes in methylation observed in response to infection are losses rather than gains in methylation, in accordance with previous findings (Klug et al. 2010).

We found that only $6 \%$ of hypomethylated regions overlapped with a promoter (Fig. 1D) and that the vast majority of hypomethylated regions were located distal to TSSs (median distance of $\sim 35 \mathrm{~kb}$ from the nearest TSS) (Fig. 1E; Supplemental Table S2). Hypomethylated regions occurred in genomic regions that show increased levels of evolutionary conservation (Supplemental Fig. S4), a finding that supports their functional importance. Moreover, gene ontology analysis revealed that these regions are significantly enriched (false discovery rate $[\mathrm{FDR}]<0.05$ ) near genes known to play a key role in the regulation of immune processes, including the regulation of transcription, signal transduction, and cell apoptosis (Fig. 1F; Supplemental Table S4). The set of genes near hypo-DMRs included virtually all of the "master regulators" of innate immune responses, including CREB5, REL, $N F K B 1, I R F 2$, and IRF4. It also included key genes involved in DC-mediated activation of $\mathrm{B}$ and $\mathrm{T}$ cells (e.g., CD83) and the regulation of cell death (e.g., BCL2).

\section{Active changes in methylation occur in regions enriched for 5-hydroxymethylcytosine}

The TET family proteins catalyze the conversion of methylated cytosine $(5 \mathrm{mC})$ to 5 -hydroxymethylcytosine $(5 \mathrm{hmC})$ and are thus key players in the process of active demethylation. To evaluate if $5 \mathrm{hmC}$ levels dynamically change in response to MTB infection (as expected if $5 \mathrm{mC}$ sites must pass through the $5 \mathrm{hmC}$ state before

\section{Genome Research}

www.genome.org 

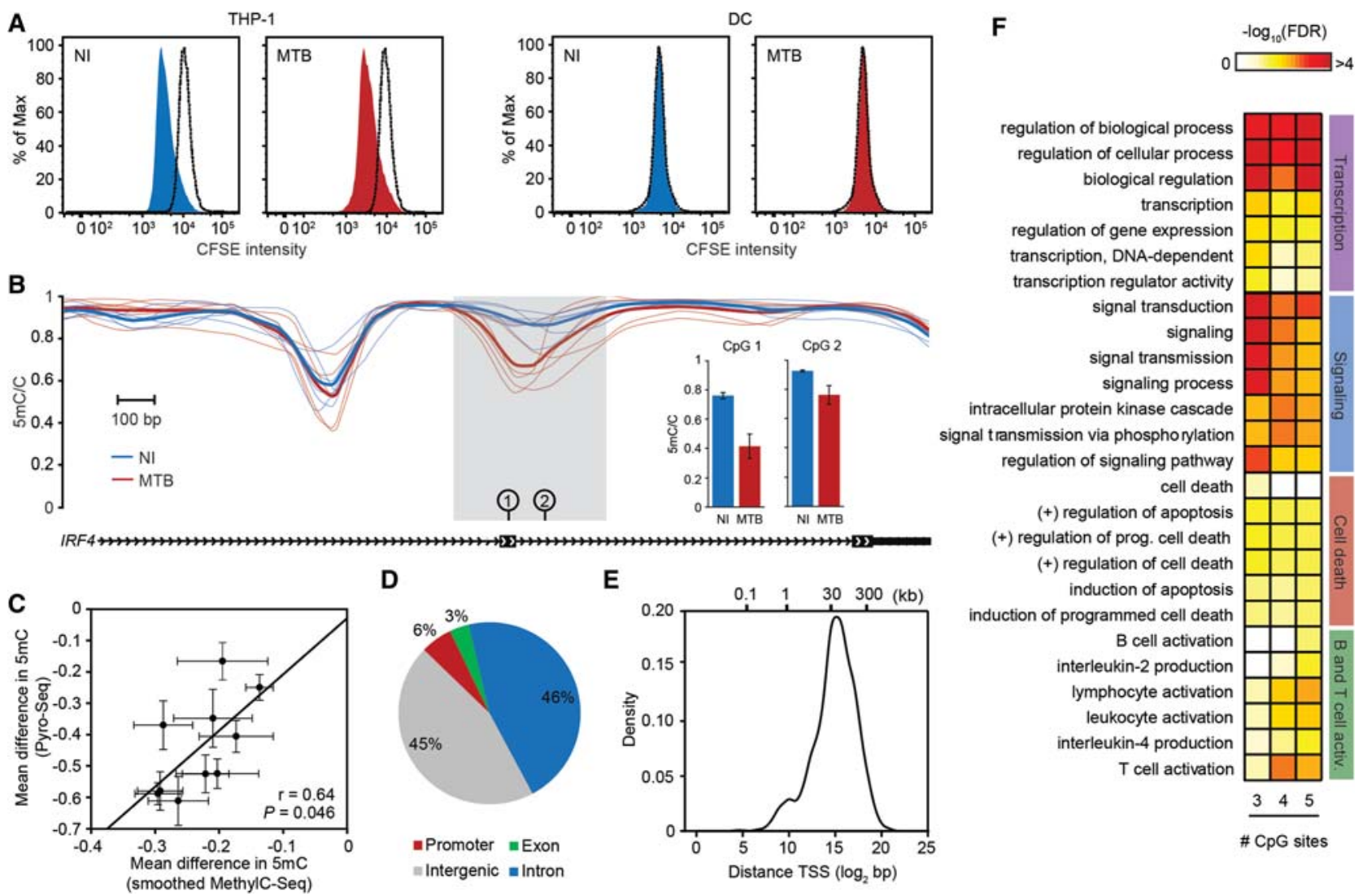

Figure 1. MTB-induced changes in methylation in post-mitotic human DCs. (A) CFSE-labeled THP-1 (left) and CFSE-labeled DCs (right). Proliferation was assayed in either noninfected cells (NI) or cells infected for $18 \mathrm{~h}$ with MTB. Similar results were observed $48 \mathrm{~h}$ post-infection (Supplemental Fig. S16). (B) Example of a region showing active loss of DNA methylation in response to MTB infection (gray shading). The plot shows smoothed methylation values ( $y$ axis) for six noninfected (blue) and six MTB-infected samples (red). Thick blue and red lines show average methylation levels for noninfected and infected cells, respectively. The inset on the right shows methylation levels at two individual $\mathrm{CpG}$ sites within the hypomethylated region using bisulfite pyrosequencing as a validation method. (C) Scatterplot showing the correlation between MethylC-seq ( $x$-axis; smoothed data) and pyrosequencing data ( $y$-axis) for mean differences in methylation between infected and noninfected cells, at $10 \mathrm{CpG}$ sites within hypomethylated DMRs. Data are represented as mean \pm SEM, $n=6$ for MethylC-seq and $n=5$ for Pyro-seq. (D) Pie chart showing the distribution of hypomethylated regions in different genomic regions. Each MTB-DMR is counted only once: The overlap of a genomic region excludes all previously overlapped MTB-DMRs, starting clockwise from promoters (TSS $\pm 500 \mathrm{bp}$; red). (E) Distribution of distances of MTB-DMRs to the nearest TSS. ( $F$ ) Representative gene ontology (GO) terms enriched among genes associated with hypomethylated regions. To demonstrate that the enriched biological processes are largely robust to the cutoff used to define MTB-DMRs, we show how these results differ depending on the number of differentially methylated CpG sites $(P<0.01)$ required to call an MTB-DMR (from at least three to at least five consecutive sites).

demethylation), we generated single base-pair resolution maps of $5 \mathrm{hmC}$ across the genome using Tet-assisted bisulfite sequencing (TAB-seq) (Yu et al. 2012) in one of the five original donors. As previously described for other cell populations (Song et al. 2011; Lister et al. 2013), we found markedly higher levels of $5 \mathrm{hmC}$ in gene bodies of highly expressed genes, consistent with a role for $5 \mathrm{hmC}$ in maintaining and/or promoting gene expression (Fig. 2A; Hahn et al. 2013; Hon et al. 2014).

Next, we evaluated if $5 \mathrm{hmC}$ marks were enriched within hypomethylated MTB-DMRs. We found that regions that became hypomethylated post-infection were already associated with significantly higher levels of $5 \mathrm{hmC}$ prior to infection (3.6-fold enrichment; Wilcoxon test; $P<1 \times 10^{-16}$ ). Upon infection, $5 \mathrm{hmC}$ levels increased even further (Wilcoxon test; $P=1.57 \times 10^{-11}$ ) (Fig. 2B,C), suggesting that $5 \mathrm{hmC}$ plays an important role in the cascade of events leading to active demethylation. The increase in $5 \mathrm{hmC}$ appears to be specific to hypomethylated regions since no enrichment was observed genome-wide, a result supported by quantitative immunocytochemistry data (Fig. 2D,E). The strik- ing enrichment of $5 \mathrm{hmC}$ within MTB-DMRs prior to infection strongly suggests that, in addition to its role as a transitory demethylation intermediate, $5 \mathrm{hmC}$ might also contribute to coordinating the gene expression program induced in response to a microbial stimulus.

MTB-DMRs overlap with enhancer elements that gain activation marks upon infection

Given that MTB-DMRs are primarily found distal to TSSs, we predicted that MTB-DMRs would overlap with enhancer regions. To test this hypothesis and evaluate how the chromatin states associated with MTB-DMRs dynamically change in response to infection, we collected ChIP-seq data for six histone marks (H3K4me1, H3K4me3, H3K27ac, H3K27me3, H3K36me3, and H3K9me3) in noninfected and infected DCs (Supplemental Table S1) from two additional donors. Using these data, we generated genome-wide, gene regulatory annotation maps for noninfected and MTB-infected DCs using the ChromHMM chromatin 

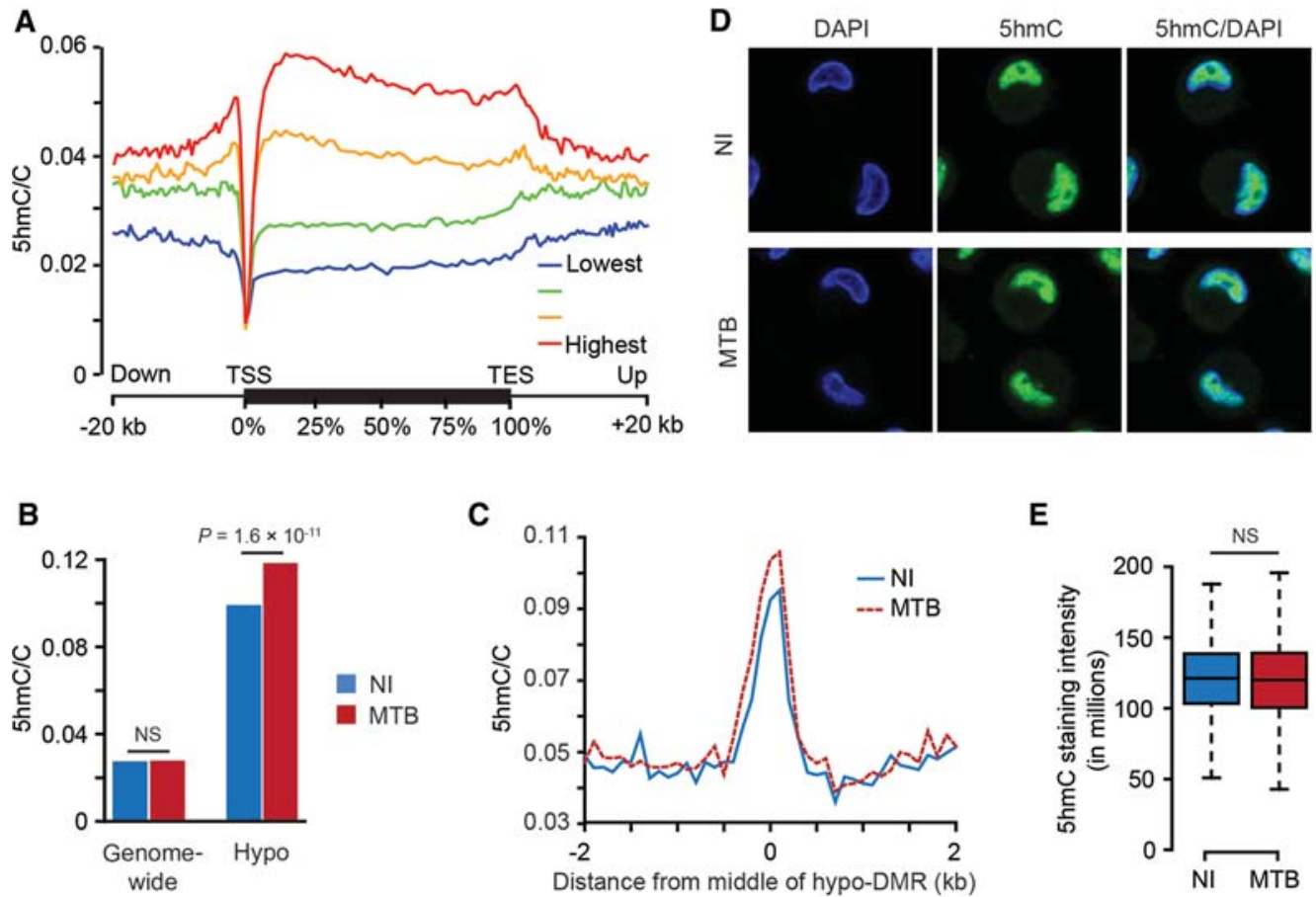

Figure 2. $5 \mathrm{hmC}$ is enriched in MTB-DMRs prior to infection. $(A)$ Metagene profiles of $5 \mathrm{hmC}$ levels relative to Ensembl transcripts expressed at different levels in human DCs. We grouped genes in four quantiles based on their expression levels in noninfected DCs. (B) Barplots showing mean $5 \mathrm{hmC} / \mathrm{C}$ ratios within hypomethylated regions, before (blue) and after infection (red). (C) Composite plots of patterns of $5 \mathrm{hmC}$ before (blue) and after (red) MTB infection $\pm 3 \mathrm{~kb}$ around the midpoint of hypomethylated regions. $(D) 5 \mathrm{hmC}$ staining in noninfected (top panel) and MTB-infected DCs (bottom panel). $5 \mathrm{hmC}$ levels are given by the levels of Alexa 488 (green: middle panel). Cells counterstained with DAPI to localize the nucleus are shown in the first panel. (E) Box plots showing the distribution of $5 \mathrm{hmC}$ staining intensity. No significant differences were observed between the two groups.

segmentation program (Fig. 3A; Supplemental Fig. S5; Ernst and Kellis 2012). We found that $41 \%$ of hypomethylated regions overlapped with a ChromHMM-annotated enhancer region (defined by the presence of H3K4me1) already present in noninfected DCs, a 7.4-fold enrichment compared to genome-wide expectations $\left(\chi^{2}\right.$ test; $P<1 \times 10^{-16}$ ) (Fig. 3B,C; Supplemental Table S2). Slightly higher enrichments ( 8 .1-fold; $P<1 \times 10^{-16}$ ) were observed when defining chromatin states in MTB-infected DCs. Given the high resolution of our histone maps, we could further distinguish between active and inactive/poised enhancer elements based on the presence or absence of the H3K27ac mark, respectively, in addition to H3K4me1 (Heintzman et al. 2007; Creyghton et al. 2010; Rada-Iglesias et al. 2011). Overall, we found that MTB infection leads to a significant increase of active enhancer elements (and decrease of inactive/poised enhancers) colocalizing with MTBDMRs (Fig. 3B,C).

We next extended our analysis by examining chromatin transition states at hypomethylated regions in response to MTB infection. We found that $42 \%$ of hypomethylated regions occurred in regions that exhibited infection-dependent changes in chromatin state, a significantly higher proportion than expected compared to the rest of the genome $\left(P_{\text {resampling }}<0.001\right)$ (Fig. 3E). The chromatin state transitions observed within hypomethylated regions were primarily explained by the acquisition of histone activating marks (e.g., H3K27ac) in MTB-infected cells. For example, among hypomethylated regions that overlapped with predefined enhancers (i.e., enhancers observable in noninfected cells), $85 \%$ of those that exhibit a change in chromatin state gained an activation mark (H3K27ac or H3K27ac+H3K4me3) (Fig. 3F,G; Supplemental Fig. S6A). This proportion was markedly larger than that observed genome-wide $(37 \%)\left(\chi^{2}\right.$ test; $P=1.1 \times 10^{-59}$ ) (Fig. 3F). Notably, we also found a large number of hypomethylated regions $(n=218$; $12.7 \%$ of all hypomethylated regions) that overlapped with heterochromatin/repressed regions before infection but gained de novo enhancer marks upon MTB infection (H3K4me1 [+H3K27ac + H3K4me3]). The number of de novo enhancers we observed among hypomethylated regions was significantly higher than expected by chance $\left(P_{\text {resampling }}<0.001\right)$ (Fig. 3D,E,G; Supplemental Fig. S6A). The identification of enhancers only present in infected DCs resembles recent findings showing that, in response to different immune stimuli, mouse macrophages can gain de novo putative enhancer regions that were absent in naive cells (Kaikkonen et al. 2013; Ostuni et al. 2013). Interestingly, we observed that $5 \mathrm{hmC}$ was significantly enriched among de novo hypo-DMRs prior to infection (Wilcoxon test; $P=5.27 \times 10^{-149}$ ), suggesting that $5 \mathrm{hmC}$ might be an early "premarking" mechanism of enhancer activation, even before the deposition of H3K4me1 marks (Supplemental Fig. S6A,B).

Finally, we found that MTB-induced activation or de novo gain of enhancer elements at hypomethylated regions was associated with the induction of putative enhancer RNAs (eRNAs) (Wang et al. 2011) in these intergenic regions (as measured by whole-transcriptome RNA-seq) as well as with increased levels of histone marks associated with transcriptional activity (Supplemental Fig. S7). Moreover, changes in eRNA levels in response to MTB infection show a striking positive correlation with changes in gene expression levels of nearby genes $\left(r=0.49, P=7.6 \times 10^{-13}\right)$ (Supplemental Fig. S7), in support of a mechanistic link between demethylation, eRNA production, and the regulation of proximal protein-coding genes (Lam et al. 2014). 
A

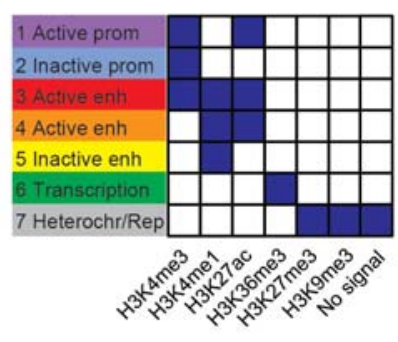

D

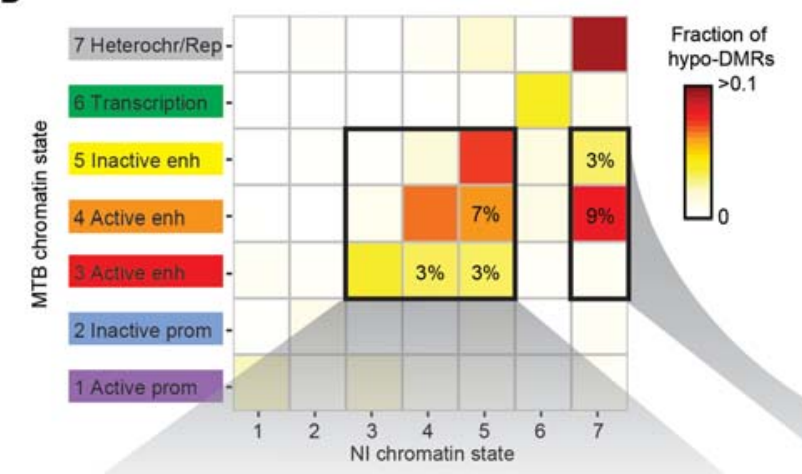

G

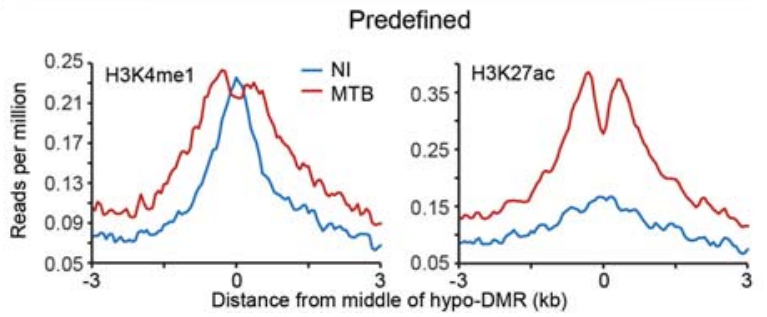

B

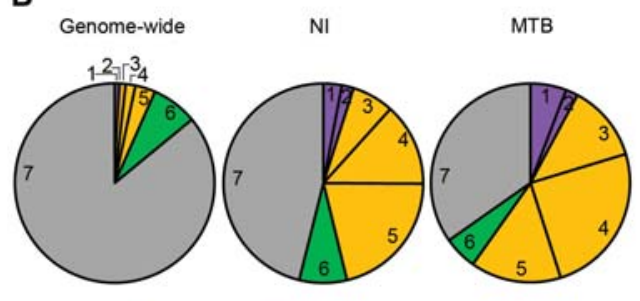

Dromoter Enhancer ETranscription 1 Heterochr/Repressed

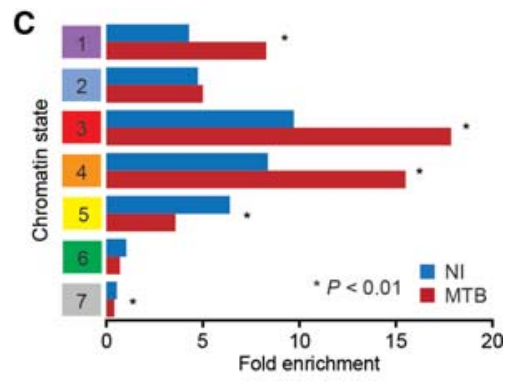

$\mathbf{F}$
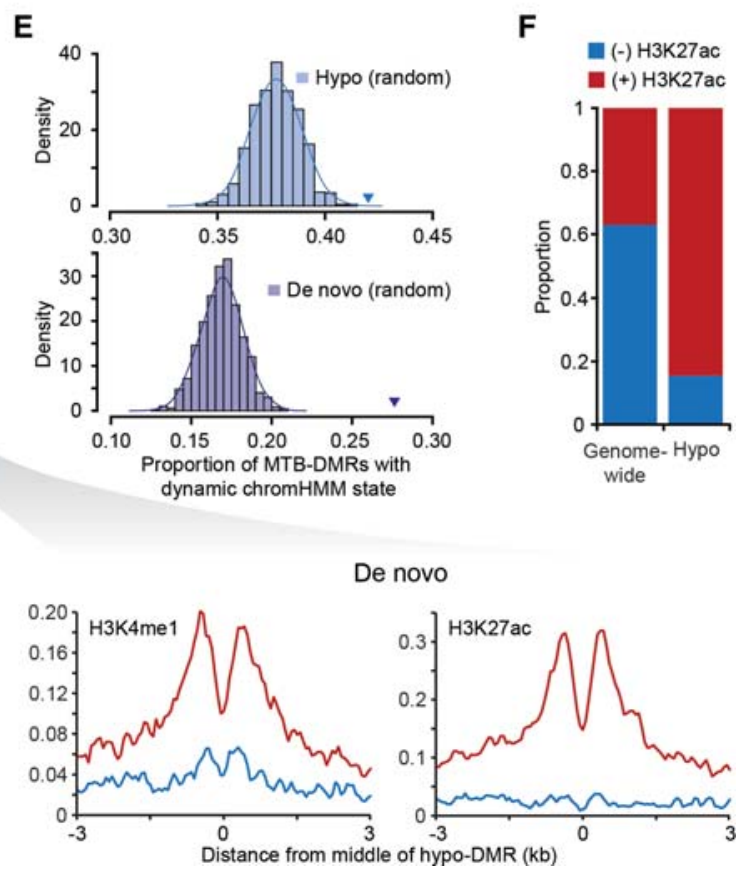

De novo

Figure 3. MTB-DMRs overlap with enhancer elements that become active upon infection in hypomethylated regions. ( $A$ ) Combination of histone patterns used to define the seven chromatin states. The precise relative contribution of each chromatin mark to each of the chromHMM-defined states can be found in Supplemental Figure S3. Note that state 7 was defined by either no signal or the presence of either H3K27me3/H3K9me3. (B) Pie charts showing the distribution of chromatin state annotations genome-wide (on noninfected cells) and within all MTB-DMRs in either noninfected (NI) or MTB-infected cells. The chromatin state codes are as defined in $A$. (C) Fold enrichments of the different chromatin states within hypomethylated regions as compared to genome-wide expectations in noninfected (blue) and MTB-infected cells (red). $(D)$ Heat map of the proportion of hypomethylated regions by chromatin transition state. The $x$-axis represents the chromatin states defined in noninfected DCs and the $y$-axis the chromatin state of the same region in MTB-infected DCs. The two bold inner boxes indicate two subgroups of hypomethylated regions, (left) predefined enhancers (detectable enhancers in noninfected DCs) and (right) de novo enhancers (detectable enhancers only in MTB-infected DCs). The numbers inside the cells refer to the proportion of hypomethylated regions that undergo each of the highlighted transitions. ( $E$ ) (Top panel) Histogram showing the observed proportion of regions that change chromatin state after infection (any transition) when sampling 1000 random sets of regions matched to the chromatin states found in noninfected samples within hypomethylated regions. Each random set contains the same number of hypomethylated regions as those identified in the true data ( $n=1714$ ). The blue triangle represents the observed proportion of hypomethylated regions that changed chromatin state in response to MTB infection. (Bottom panel) Same as above but focusing on regions of the genome labeled as heterochromatin/repressed before infection (state 7 ; $n=790$ ) that gain de novo enhancer marks upon MTB infection (states 3, 4, or 5). The purple triangle represents the proportion observed within the true set of hypomethylated regions. $(F)$ Bar plot showing the proportion of hypomethylated regions that overlap with enhancers and show dynamic changes in chromatin state, as defined by the gain or loss of $\mathrm{H} 3 \mathrm{~K} 27 \mathrm{ac}$ mark. (G) Composite plots of patterns of H3K4me1 and H3K27ac ChIP-seq signals \pm 3 kb around the midpoints of hypomethylated regions ( $x$-axis) overlapping with predefined (left) and de novo (right) enhancers.

\section{MTB-DMRs are bound by signal-dependent transcription factors}

We next asked if MTB infection was associated with changes in the levels of chromatin accessibility in MTB-DMRs. We mapped regions of open chromatin in noninfected and infected DCs based on genome-wide sequencing of regions showing high transposase (Tn5) sensitivity (using ATAC-seq in one additional donor) (Buenrostro et al. 2013). Overall, we observed that MTB-DMRs colocalize with regions of open chromatin, which further reinforces the regulatory potential of these regions (Fig. 4A). Interestingly, we found that the response to MTB infection was accompanied by a striking increase in Tn5 sensitivity levels in hypomethylated regions, which indicates that the chromatin in these regions became more accessible after infection (Fig. 4A). This observation is commensurate with our data showing the acquisition of active histone marks in these regions and further supports the idea that hypomethylated regions frequently reflect the presence of regulatory elements that become more active in response to infection.

An attractive feature of ATAC-seq data is the ability to identify motif instances occupied by transcription factors (TFs) within 
A

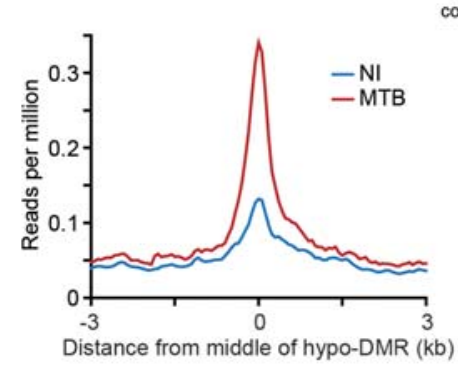

C

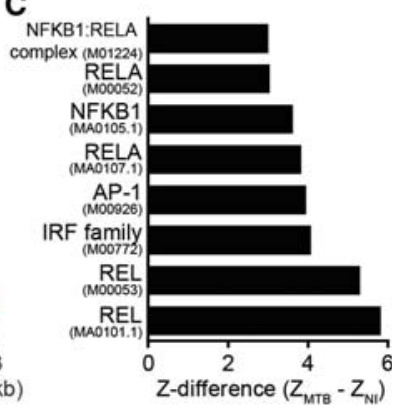

B

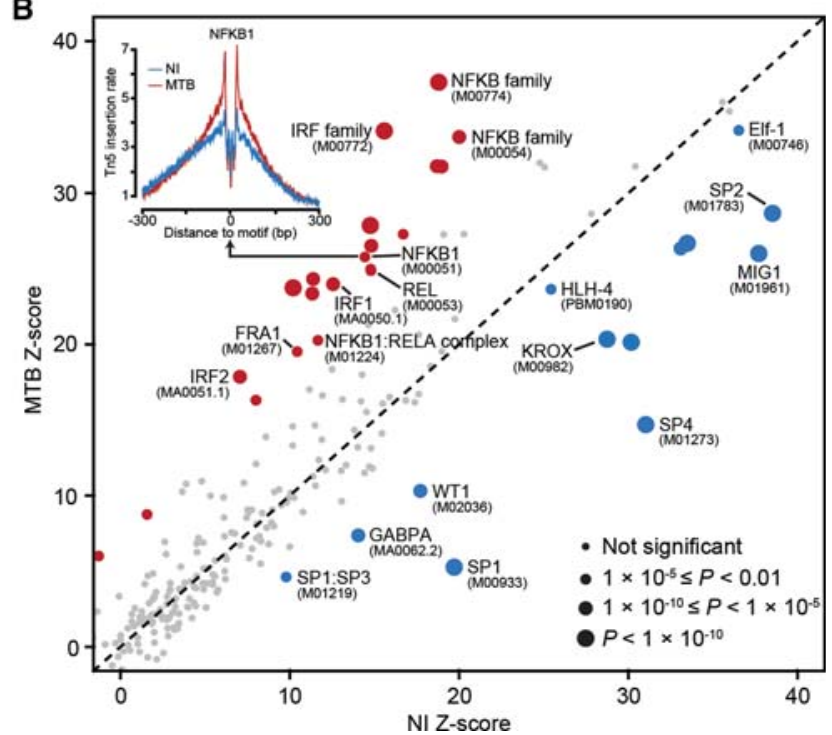

Figure 4. MTB-DMRs are bound by signal-dependent transcription factors. (A) Tn5-accessibility profiles before $(\mathrm{NI})$ and after MTB infection, \pm 3 $\mathrm{kb}$ around the midpoints of hypomethylated regions. (B) Scatterplot comparing transcription factor occupancy score predictions between noninfected ( $x$-axis) and MTB-infected DCs ( $y$-axis). The size of the dots is proportional to the level of statistical significance supporting differential binding in response to MTB infection. Red dots represent TFs that show evidence for increased binding after MTB infection, and blue dots represent TFs that show evidence for decreased binding after infection. The inset on the top left corner shows the genome-wide footprint of the NF-kB ( $\mathrm{p50}$ motif (motif ID: M00051) in noninfected (blue) and MTB-infected DCs (red). In this example, the footprint in MTB-infected DCs is clearly stronger, which supports increased TF binding to the NF-kB (p50) motif genome-wide, upon MTB infection. (C) TF motifs (motif IDs in parentheses) that show significantly increased binding in hypomethylated regions after MTB infection.

regions of open chromatin (Neph et al. 2012; Buenrostro et al. 2013). We did so by using a modified version of the Centipede algorithm (Pique-Regi et al. 2011) specifically devised to test for aggregate differential binding of TFs between two experimental conditions. This method, which we call CentiDual, compares the intensity of the Tn5 sensitivity-based footprint across all matches to a given motif in the genome, between noninfected and infected samples (see Methods for details on the statistical model). We found compelling evidence for measurable, genome-wide transcription factor activity (i.e., binding to the genome; Bonferronicorrected $P<0.05$ ) in either noninfected or infected DCs for 264 TF binding motifs, representing over 200 unique transcription factors (some TFs can bind different motifs) (Supplemental Table S5). Of these TF binding motifs, we found 55 that were differentially bound between noninfected and infected DCs (Bonferroni-corrected $P<0.05 ; 27$ show increased binding and 28 show decreased binding) (Fig. 4B). Among TF binding motifs showing increased genome-wide binding after infection, we found several that are associated with NF-kB/Rel (e.g., NFKB1, REL) and IRF (e.g., IRF1, IRF2) family members (Fig. 4B; Supplemental Table S5), both of which play a primary role in the regulation of inflammatory signals in response to infection (Smale 2010). Interestingly, several CTCF motifs showed significantly decreased binding in infected DCs (Bonferroni-corrected $P<1.85 \times 10^{-14}$ ) (Supplemental Table S5). CTCF is a well-established transcriptional insulator (Ong and Corces 2014), raising the possibility that the release of CTCF in response to infection might be an important mechanism for the regulation of efficient immune responses.

We next used CentiDual to test for differential binding within MTB-DMRs. Within hypomethylated regions, we found increased binding $(\mathrm{FDR}<0.1)$ at eight TF binding motifs after infection. Strikingly, all of these motifs were associated with immune-induced TFs from the NF- $\mathrm{B} /$ Rel (e.g., REL; FDR $=1.57 \times 10^{-6}$ ), AP-1 $\left(\mathrm{FDR}=4.9 \times 10^{-3}\right)$, or IRF $\left(\mathrm{FDR}=3.97 \times 10^{-3}\right)$ families (Fig. $4 \mathrm{C}$; Supplemental Table S5). This result demonstrates that hypomethyated regions correspond to places where immune-activated TFs are recruited after infection. In accordance with this argument, we found that, in infected DCs, TF binding motifs associated with NF-kB/Rel, AP-1, and IRF families were all significantly enriched within hypomethylated regions (up to 16-fold) (Supplemental Fig. S8A). Indeed, in MTB-infected DCs, over 50\% of the hypomethylated regions were bound by at least one of these signal-dependent TFs, which corresponds to a 3.8-fold increase relative to chance expectations (based on sampling random regions of the genome matched for length and GC content; $\chi^{2}$ test; $P=3.94 \times 10^{-63}$ ) (Supplemental Fig. S8B).

\section{MTB-DMRs are associated with genes differentially expressed in response to $M T B$ infection}

Finally, we asked if genes associated with hypomethylated regions were more likely to change expression levels in response to infection. We classified 2051 and 1947 genes as significantly up- or down-regulated post-infection, respectively (FDR $<1 \times 10^{-4}$ and $\mid \log _{2}$ fold change| $>1$ ) (Supplemental Table S6). We next tested whether genes located near hypomethylated regions were more likely to be differentially expressed upon MTB infection relative to all genes in the genome. To do so, we first associated each hypomethylated region with a unique gene using the following criteria: If a hypomethylated region was located within a gene body, the region was assigned to that gene; otherwise, we assigned each hypomethylated region to the gene with the TSS closest to the midpoint of the MTB-DMR. Then, we tested for an enrichment of differentially expressed (DE) genes among three classes of genes: (1) "hypo-DMR-genes" corresponding to the set of genes associated with hypomethylated regions $(n=1291)$; (2) "predefined-DMRgenes" corresponding to the set of genes in hypomethylated regions that overlapped with predefined enhancer elements $(n=$ 508 , a subset of class 1); and (3) "de novo-DMR-genes" corresponding to the set of genes in hypomethylated regions that overlapped with de novo enhancer elements $(n=180$, also a subset of class 1$)$.

We found that hypo-DMR-genes (class 1 ) were significantly enriched among DE genes (1.6-fold, $\chi^{2}$-test; $P=1.07 \times 10^{-17}$ ) (Fig. $5 \mathrm{~A}, \mathrm{~B})$ compared to all genes in the genome, consistent with the observation that changes in DNA methylation were globally correlated to changes in expression after infection (Supplemental Fig. S9). 
A

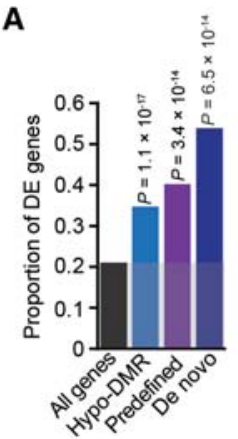

B

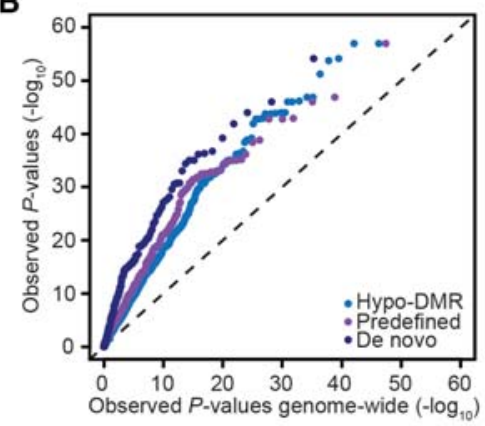

C

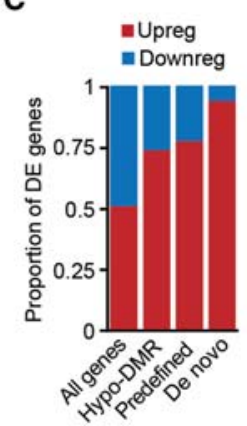

D

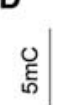

怘

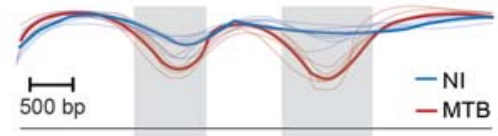

\begin{tabular}{l|l}
$\mathrm{O}$ & $\mathrm{NI}$ \\
$\stackrel{\mathrm{E}}{\mathrm{\omega}}$ & $\mathrm{MTB}$
\end{tabular}

\begin{tabular}{l|l}
$\bar{\Phi}$ & $\mathrm{NI}$ \\
$\frac{\Sigma}{2}$ & MTB
\end{tabular}

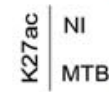

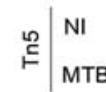

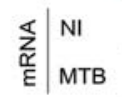

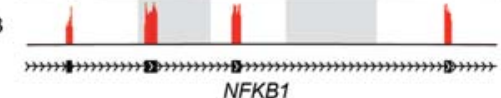

E
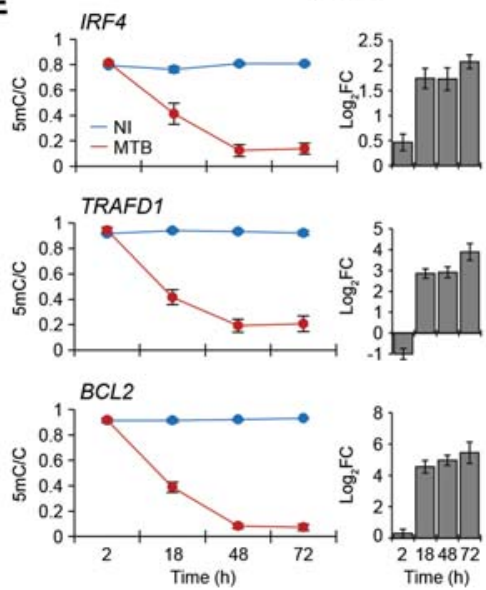
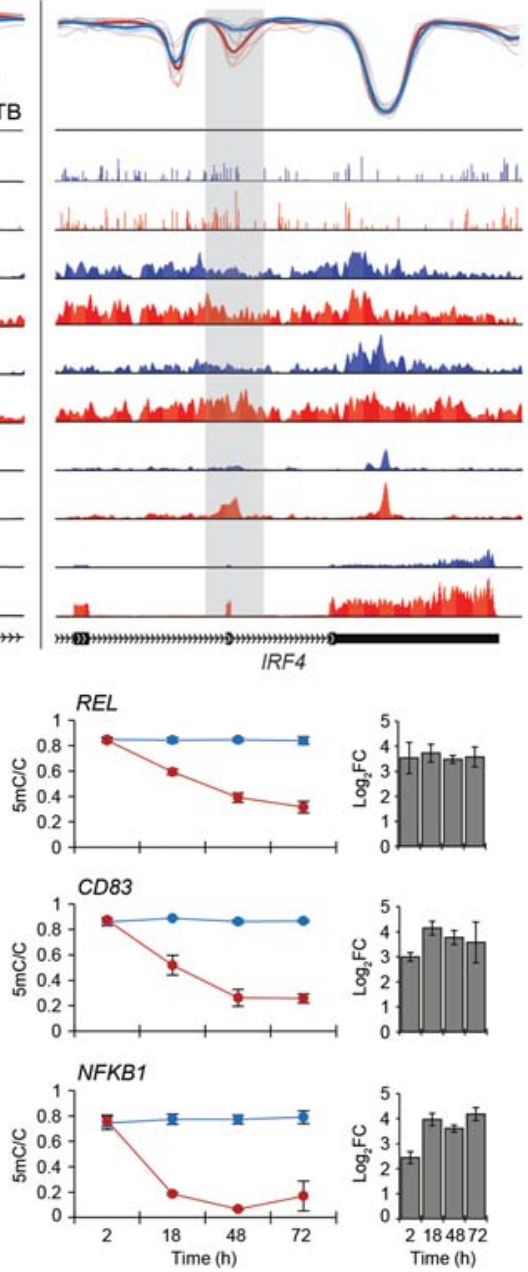

Figure 5. Differential methylation is coupled to differential gene expression. $(A)$ Proportion of differentially expressed (DE) genes ( $y$-axis) observed among all tested genes and among genes associated with different subgroups of hypo-DMRs. (B) QQ-plot showing that genes in the vicinity of hypo-DMRs show stronger statistical evidence for being differentially expressed in response to MTB infection ( $P$-values on $y$ axis) compared to all genes tested ( $P$-values on $x$-axis). (C) Proportion of up- and down-regulated genes among DE genes associated with the different subgroups of hypo-DMRs. (D) Examples of genes encoding for two key transcription factors, NFKB1 (left panel) and IRF4 (right panel) that are strongly up-regulated in response to MTB infection and for which we identified one or more hypomethylated regions (gray shading) that overlap with putative enhancer elements. Normalized read signals for the indicated features are shown for noninfected (blue tracks) and infected conditions (red tracks). (K4me1) H3K4me1, (K27ac) H3K27ac, (Tn5) transposase-accessible chromatin, (mRNA) mRNA expression levels. $(E)$ Changes in DNA methylation levels ( $y$-axis) measured by pyrosequencing across four time points after MTB infection $\left(2,18,48\right.$, and $72 \mathrm{~h}$ ) along with the corresponding fold changes in $\log _{2}$ scale $\left(\log _{2} \mathrm{FC}\right)$ in normalized gene expression of the associated gene. Blue and red lines represent average methylation levels in noninfected and MTB-infected DCs, respectively. All data are represented as mean \pm SEM, with a minimum of three biological replicates per group. PyroMark and real-time PCR data are reported in Supplemental Tables S7 and S8, respectively.

This enrichment was noticeably stronger for predefined-DMR-genes (class 2; 1.9 fold, $P=3.37 \times 10^{-14}$ ) and even more so for de novo-DMR-genes (class $3 ; 2.5$ fold, $\left.P=6.52 \times 10^{-14}\right)$. Indeed, among de novo-DMR-genes, $54 \%$ were $\mathrm{DE}$, even at the very stringent cutoffs we used to define DE genes (Fig. 5A,B). Among DE genes associated with hypomethylated regions, $74 \%$ were up-regulated after MTB infection-substantially more than the $51 \%$ of up-regulated genes observed genome-wide $\left(\chi^{2}\right.$-test; $P=4.4 \times$ $10^{-79}$ ) (Fig. 5C,D). This observation was even more pronounced when focusing specifically on predefined-DMR-genes (class 2) and de novo-DMR-genes (class $3)$, for which $78 \%\left(P=8.68 \times 10^{-36}\right)$ and $94 \%\left(P=6.9 \times 10^{-23}\right)$, respectively, were associated with increased expression levels in response to infection (Fig. 5C).

Finally, we performed a paired timecourse analysis of gene expression and DNA methylation levels for six hypoDMRs (associated with six immune-related genes: IRF4, REL, TRAFD1, CD83, $B C L 2, N F K B 1)$ aimed at defining the relative order of changes in DNA methylation versus changes in gene expression. We found that differential methylation was undetectable at $2 \mathrm{~h}$ post-infection, even though half of the genes associated with these sites (three out of the six genes tested) were already significantly up-regulated (Fig. 5E). Thus, we speculate that demethylation is not required for $\mathrm{TF}$ binding at enhancer elements or for the subsequent up-regulation of the associated gene. However, for all CpG sites tested, the magnitude of DNA hypomethylation (relative to noninfected cells) increased at later time points until methyl marks were almost completely erased in infected cells, even if for most genes the largest fold changes in gene expression were observed at earlier time points (Fig. 5E).

\section{Discussion}

The possibility that active changes in methylation, particularly demethylation, can occur in mammals has been a matter of debate for decades (Ooi and Bestor 2008; Pastor et al. 2013). Here, we provide compelling evidence that the response of human DCs to MTB infection is accompanied by widespread, rapid loss in DNA methylation. Although many possible mechanisms can account for these losses (Kohli and Zhang 2013), the observation that hypomethylated regions show increased levels of $5 \mathrm{hmC}$ in 
response to MTB infection strongly suggests that the family of TET proteins (TET1, TET 2, and TET3) is involved in this process. This possibility is further supported by recent studies showing that TET2 is required for active DNA demethylation in human monocytes (Klug et al. 2013) and during brain development (Lister et al. 2013). TET2 is also highly expressed in DCs at both the mRNA and protein levels (Supplemental Fig. S10).

By integrating our methylation maps with ChIP-seq data on six histone marks, we show that active demethylation occurs almost exclusively at distal regulatory elements, particularly enhancers. This observation, which is robust to the cutoffs used to call MTB-DMRs (Supplemental Fig. S11), parallels what has been previously described in differentiating cells and during developmental processes (Ji et al. 2010; Stadler et al. 2011; Ziller et al. 2013) despite the fact that the mechanisms controlling active and passive changes in methylation are markedly different (Kohli and Zhang 2013). In contrast to previous studies that also reported active changes in methylation (e.g., in response to neuronal activation [Guo et al. 2011], or during monocyte differentiation into macrophages or dendritic cells [Klug et al. 2010]), we found a strong association between DMRs and changes in gene expression of nearby genes. The apparent discrepancy between our results and those previously reported is probably explained by the fact that past studies have only investigated active methylation changes in promoter regions, which our data suggest are infrequent, or only on a small subset of all CpG sites in the (mouse) genome ( 1\%). Moreover, we decided to focus on differentially methylated regions (three or more consecutive differentially methylated CpGs) instead of methylation changes at individual CpG sites (Klug et al. 2010; Guo et al. 2011), a decision that likely enriched our analysis for DMRs involved in changes in gene expression. In support of this hypothesis, we found that the enrichment for DE genes becomes stronger as we focus on MTB-DMRs with a larger number of differently methylated CpG sites (Supplemental Fig. S11). More broadly, our results highlight the key importance of using single base-pair resolution maps of the DNA methylome in order to fully capture the relationship between changes in methylation and changes in gene expression.

We show that demethylation is often associated with the gain of histone activation marks and the recruitment of immune-activated TFs in response to infection. The recruitment of NF- $\mathrm{BB}$ and other master regulators to hypomethylated regions is likely associated with the opening of the chromatin in these regions, although it remains unclear whether the chromatin opens to allow the binding of these TFs (i.e., prior to binding) or if the observed increase in chromatin accessibility is a consequence of the binding itself. Our results shed some light on this problem. Specifically, we observed that changes in gene expression sometimes tend to occur prior to detectable changes in DNA methylation, at least in the hypoDMRs we investigated in our time-course experiment. These results support a model in which TF binding to enhancers leads to gene up-regulation followed by active demethylation, rather than vice versa, consistent with the sequence of events proposed for other cellular contexts (Stadler et al. 2011; Schubeler 2015). We note, however, that our pyrosequencing data do not allow us to distinguish between $5 \mathrm{mC}$ and $5 \mathrm{hmC}$. Thus, it is possible that $5 \mathrm{hmC}$ levels were increased $2 \mathrm{~h}$ post-infection and that these changes preceded the activation of certain enhancers, as recently suggested in T cells (Ichiyama et al. 2015). More generally, we cannot completely exclude the possibility that demethylation occurred prior to changes in gene regulation but only in a small proportion of the cells $(1 \%-2 \%$, based on the sensitivity threshold of pyrosequencing assays) (Tost and Gut 2007), making it difficult to detect at the 2 -h time point. Under this scenario, demethylation in only a few cells could account for the observed changes in gene expression. However, we have previously shown that $>30 \%$ of DCs uptake MTB bacteria after only $1 \mathrm{~h}$ post-infection using the same protocol (Barreiro et al. 2012), and in single-cell RNA-seq data from dendritic cells, $>50 \%$ are transcriptionally responsive to immune challenge (Shalek et al. 2014). Thus, it seems unlikely that our results reflect a response driven by only a small minority of cells. Importantly, even if TF binding instigates changes in methylation, binding alone is not sufficient; the vast majority (>99\%) of binding events induced by infection occur at regions that do not change methylation (Supplemental Fig. S12).

Finally, there is increasing evidence that, after a first encounter with a pathogen or other immune stimulus, innate immune cells keep such attacks "in memory." As a result, they are able to mount faster and stronger gene transcriptional responses upon restimulation and exhibit increased resistance to secondary infection. This process, termed trained immunity (Monticelli and Natoli 2013; Quintin et al. 2014; Saeed et al. 2014), has been attributed to epigenetic reprograming at the level of histone H3 methylation based on the observation that distal regulatory elements that gain de novo H3K4me1 (i.e., de novo enhancer marks) in response to immune activation generally do not lose this mark after the stimulation has ceased (Ostuni et al. 2013). Although epigenetic programming through histone modifications might be an important factor in trained immunity, our results raise the possibility that changes in DNA methylation might also contribute to short-term memory in innate immune cells. Indeed, changes in DNA methylation might be ideally suited as a mechanism of epigenetic memory since these changes are expected to be thermodynamically more stable and longer lasting than changes in histone marks. In support of this idea, we observed that the magnitude of DNA hypomethylation gradually increased with time since infection and never reverted back to higher levels during our 72-h time-course experiment. Moreover, we show that the gain of de novo enhancers-assumed to account for trained immunity-often occurs concomitantly with the loss of DNA methylation in the same regions. Our results thus raise the possibility that trained immunity might not only be due to post-transcriptional changes in histone marks but also, and possibly primarily, due to changes in DNA methylation.

\section{Methods}

\section{Biological material and sequencing libraries}

Details of the experimental and statistical procedures can be found in the Supplemental Methods section. Blood samples from healthy donors were obtained from the Indiana Blood Center. A signed written consent was obtained from all of the participants and the project was approved by the ethics committee at the CHU Sainte-Justine (protocol \#4023). Blood mononuclear cells from each donor were isolated by Ficoll-Paque centrifugation, and blood monocytes were purified from peripheral blood mononuclear cells (PBMCs) by positive selection with magnetic CD14 MicroBeads (Miltenyi Biotec). Monocytes were then derived into DCs as previously described (Barreiro et al. 2012) and subsequently infected with MTB for $18 \mathrm{~h}$ at a multiplicity of infection of 1-to-1. For the ChIP-seq and ATAC-seq experiments, we used heat-killed bacteria (5-to-1 ratio), which leads to virtually the same transcriptional response at $18 \mathrm{~h}$ as that observed with live MTB $(r=0.91)$ (Supplemental Fig. S13). RNA-seq libraries were prepared using 
the TruSeq RNA Sample Prep Kit v2 or the Illumina Total Stranded RNA Library kit, as per the manufacturer's instructions. MethylCseq libraries were generated by ligation of methylated sequencing adapters to fragmented genomic DNA, followed by gel purification, sodium bisulfite conversion, and six cycles of PCR amplification. TAB-seq libraries were generated as previously described $(\mathrm{Yu}$ et al. 2012), and ChIP-seq libraries for the six histone marks were prepared following the Illumina protocols, with minor modifications (see Supplemental Methods). ChIP-seq signals from the two biological replicates were highly concordant (mean $r=0.94$ and range $=0.87-0.99$ ) (Supplemental Fig. S14) and were combined for all analysis. Finally, ATAC-seq libraries were generated from 100,000 cells, as previously described (Buenrostro et al. 2013). Sequencing was performed using the Illumina HiSeq 2000 or 2500 , as per the manufacturer's instructions.

\section{CFSE proliferation assay}

DCs and THP-1 cells were covalently labeled with carboxyfluorescein diacetate succinimidyl ester (Life Technologies) as described in detail elsewhere (Quah and Parish 2010). Briefly, cells were washed with PBS and resuspended with $5 \mathrm{mM}$ CFSE. After a 5-min incubation at room temperature, cells were thoroughly washed with PBS containing 5\% FCS before plating in complete culture medium.

\section{5hmC staining}

The protocol was adapted from Santos et al. (2003). DCs were cultured on poly-L-lysine-coated coverslips and fixed for $30 \mathrm{~min}$ in $4 \%$ paraformaldehyde in PBS and permeabilized with $0.2 \%$ Triton X-100 in PBS for $30 \mathrm{~min}$ at room temperature (RT). Cells were then washed with $0.05 \%$ Tween 20 in PBS and were treated with $1 \mathrm{M} \mathrm{HCl}$ plus $0.1 \%$ Triton X-100. After $30 \mathrm{~min}$ at $37^{\circ} \mathrm{C}$, cells were incubated with $100 \mathrm{mM}$ Tris/ $\mathrm{HCl}(\mathrm{pH} 8.5)$ for $30 \mathrm{~min}$ and blocked for $2 \mathrm{~h}$ in PBS with 1\% BSA, $0.05 \%$ Tween-20, and 2\% goat serum. Cells were incubated with 5-hydroxymethylcytosine antibody (ActiveMotif), followed by Alexa 488 goat anti-rabbit antibody (Life Technologies) for $1 \mathrm{~h}$ at RT. The slides were mounted with Fluoromount $\mathrm{G}$ (SouthernBiotech), and cells counterstained with DAPI to localize the nucleus. A laser-scanning microscope (Zeiss LSM 700) in the tile scan mode was used to capture a mosaic of images. Fluorescence was quantified using Fiji software. Average fluorescence estimates were calculated from 1769 noninfected cells and 1532 MTB-infected cells.

\section{Read processing and alignment}

Sequencing data were processed using the Illumina analysis pipeline, and FASTQ format reads were aligned to the human reference genome (GRCh37/hg19) using Bowtie 2 (Langmead and Salzberg 2012). Methylation levels for each CpG site were estimated by counting the number of sequenced C ("methylated" reads) divided by the total number of reported $\mathrm{C}$ and $\mathrm{T}$ ("unmethylated" reads) at the same position of the reference genome using the Bismark package (Krueger and Andrews 2011). The summarized methylation estimates of strand-merged $\mathrm{CpG}$ sites were used to identify differences in methylation between noninfected and infected samples using the R package BSmooth (Hansen et al. 2012). RNA-seq-based gene expression levels were estimated using HTSeq and differently expressed genes following MTB infection of DCs using the R package DESeq2 (Anders et al. 2013). ChromHMM (Ernst and Kellis 2012) was used to segment the genome into different chromatin states based on six histone modifications and the ChIP input. The ChromHMM model was learned separately for both infected and noninfected DCs (main text) or using a unified model that learns and defines chromatin states in both infected and noninfected DCs at the same time (Supplemental Fig. S15).

\section{ATAC-seq data processing and footprinting analysis}

Footprinting analyses were performed using a modified version of the Centipede algorithm (Pique-Regi et al. 2011) specifically devised to test for differential binding between two experimental conditions. To determine which TFs were active in the first step, we calculate a $Z$-score corresponding to the PWM effect in the prior probability in Centipede's logistic model, and we determined as active those that had a Bonferroni-corrected $P<0.05$. The $Z$-score corresponds to the $\beta$ parameter in:

$$
\log \left(\frac{\pi_{l}}{1-\pi_{l}}\right)=\alpha+\beta \text { PWMscore }_{l},
$$

where $\pi_{1}$ represents the prior probability of binding in Centipede's model in motif location $l$. In the second step, we first trained Centipede assuming that the footprint was bound in the two conditions. Then, we fixed the model parameters and generated a likelihood ratio and posterior probability $\pi_{\mathrm{lt}}$ for each condition $t$ separately and for each site $l$. To detect if the footprint was more active in one of the two conditions, we fit a logistic model that included an intercept for each condition ( $\alpha$ and $\delta$ ), the PWM effect $\beta$, and PWM times the treatment effect $\gamma$ :

$$
\begin{aligned}
\log \left(\frac{\pi_{l t}}{1-\pi_{l t}}\right)= & \alpha \times\left(1-I_{t}\right)+\beta \times \text { PWMscore }_{l}+\delta \times I_{t}+\gamma \\
& \times\left(I_{t} \times \text { PWMscore }_{l}\right),
\end{aligned}
$$

where $I_{t}$ is an indicator variable that takes the value 1 if $t=$ "treatment" and 0 if $t=$ "control." We then calculated a $Z$-score for the interaction effect $\gamma$, corresponding to the evidence for conditionspecific binding.

\section{Data visualization in the Immune Epigenome Browser}

The browser, implemented using the WashU Epigenome Browser web interface (Zhou and Wang 2012), can be accessed at http:// luis-barreirolab.org/EpigenomeBrowser. Along with RefSeq gene annotations, it includes 25 data tracks showing (1) the genomic location of MTB-DMRs, (2) smoothed site-specific $5 \mathrm{mC}$ values, (3) $5 \mathrm{hmC}$ values, (4) all histone mark ChIP-seq read signals (H3K4me3, H3K4me1, H3K27ac, H3K27me3, H3K36me3, and H3K9me3), (5) Tn5-transposase (i.e., chromatin accessibility) read signals, (6) mRNA read signals, and (7) predicted binding sites for the 55 transcription factors that significantly change genomewide binding levels in response to MTB infection. All data sets are shown for both noninfected (NI) and MTB-infected (MTB) conditions with respect to the GRCh37/hg19 reference sequence. Note that, for ease of visualization, several tracks are not shown under default parameters. These can be added by going to: Tracks $\rightarrow$ Custom tracks $\rightarrow$ List of all.

\section{Data access}

Data generated in this study have been submitted to the NCBI Gene Expression Omnibus (GEO; http://www.ncbi.nlm.nih. gov/geo/) under accession numbers GSE64173 (ATAC-seq), GSE64175 (ChIP-seq), GSE64181 (TAB-seq), GSE64182 (wtRNAseq), GSE64179 (mRNA-seq), and GSE64177 (MethylC-seq). PyroMark and real-time PCR data are reported in Supplemental Tables S7 and S8, respectively. 


\section{Acknowledgments}

We thank B. Jabri, V. Abadie, and J.F. Brinkworth for helpful discussions and comments on the manuscript; K. Michelini and C. Chavarria for technical assistance running the sequencer; G. Stewart for the gift of the MTB strain used in this study; and P. Roux for advice on the confocal microscopy. We thank Calcul Quebec and Compute Canada for managing and providing access to the supercomputer Briaree from the University of Montreal. This study was funded by National Institutes of Health (NIH) Grant AI087658 (to Y.G. and L.T.), by grants from the Canadian Institutes of Health Research (301538 and 232519), the Human Frontiers Science Program (CDA-00025/2012), and the Canada Research Chairs Program (950-228993) (to L.B.B.), by the Canadian Institutes of Health Research/Canadian Epigenetics, Environment and Health Research Consortium (to T.P.), and by the NIH grant HG006827 (to C.H.). M.S.K. is a Canada Research Chair in Social Epigenetics and a Senior Fellow of the Canadian Institute for Advanced Research. A.P. was supported by a fellowship from the Réseau de Médecine Génétique Appliquée (RMGA).

\section{References}

Anders S, McCarthy DJ, Chen Y, Okoniewski M, Smyth GK, Huber W, Robinson MD. 2013. Count-based differential expression analysis of RNA sequencing data using $\mathrm{R}$ and Bioconductor. Nat Protoc 8: 1765-1786.

Ardeshna KM, Pizzey AR, Thomas NS, Orr S, Linch DC, Devereux S. 2000. Monocyte-derived dendritic cells do not proliferate and are not susceptible to retroviral transduction. Br J Haematol 108: 817-824.

Barreiro LB, Tailleux L, Pai AA, Gicquel B, Marioni JC, Gilad Y. 2012. Deciphering the genetic architecture of variation in the immune response to Mycobacterium tuberculosis infection. Proc Natl Acad Sci 109. 1204-1209.

Bierne H, Hamon M, Cossart P. 2012. Epigenetics and bacterial infections. Cold Spring Harb Perspect Med 2: a010272.

Bruniquel D, Schwartz RH. 2003. Selective, stable demethylation of the interleukin-2 gene enhances transcription by an active process. Nat Immunol 4: 235-240.

Buenrostro JD, Giresi PG, Zaba LC, Chang HY, Greenleaf WJ. 2013. Transposition of native chromatin for fast and sensitive epigenomic profiling of open chromatin, DNA-binding proteins and nucleosome position. Nat Methods 10: 1213-1218.

Creyghton MP, Cheng AW, Welstead GG, Kooistra T, Carey BW, Steine EJ, Hanna J, Lodato MA, Frampton GM, Sharp PA, et al. 2010. Histone H3K27ac separates active from poised enhancers and predicts developmental state. Proc Natl Acad Sci 107: 21931-21936.

Dowen RH, Pelizzola M, Schmitz RJ, Lister R, Dowen JM, Nery JR, Dixon JE, Ecker JR. 2012. Widespread dynamic DNA methylation in response to biotic stress. Proc Natl Acad Sci 109: E2183-E2191.

Ernst J, Kellis M. 2012. ChromHMM: automating chromatin-state discovery and characterization. Nat Methods 9: 215-216.

Ghisletti S, Barozzi I, Mietton F, Polletti S, De Santa F, Venturini E, Gregory L, Lonie L, Chew A, Wei CL, et al. 2010. Identification and characterization of enhancers controlling the inflammatory gene expression program in macrophages. Immunity 32: 317-328.

Guo JU, Ma DK, Mo H, Ball MP, Jang MH, Bonaguidi MA, Balazer JA, Eaves HL, Xie B, Ford E, et al. 2011. Neuronal activity modifies the DNA methylation landscape in the adult brain. Nat Neurosci 14: 1345-1351.

Hahn MA, Qiu R, Wu X, Li AX, Zhang H, Wang J, Jui J, Jin SG, Jiang Y, Pfeifer GP, et al. 2013. Dynamics of 5-hydroxymethylcytosine and chromatin marks in mammalian neurogenesis. Cell Rep 3: 291-300.

Hansen KD, Langmead B, Irizarry RA. 2012. BSmooth: from whole genome bisulfite sequencing reads to differentially methylated regions. Genome Biol 13: R83.

Hansen KD, Sabunciyan S, Langmead B, Nagy N, Curley R, Klein G, Klein E, Salamon D, Feinberg AP. 2014. Large-scale hypomethylated blocks associated with Epstein-Barr virus-induced B-cell immortalization. Genome Res 24: 177-184.

Heintzman ND, Stuart RK, Hon G, Fu Y, Ching CW, Hawkins RD, Barrera LO, Van Calcar S, Qu C, Ching KA, et al. 2007. Distinct and predictive chromatin signatures of transcriptional promoters and enhancers in the human genome. Nat Genet 39: 311-318.

Hon GC, Song CX, Du T, Jin F, Selvaraj S, Lee AY, Yen CA, Ye Z, Mao SQ Wang BA, et al. 2014. $5 \mathrm{mC}$ oxidation by Tet 2 modulates enhancer activ- ity and timing of transcriptome reprogramming during differentiation. Mol Cell 56: 286-297.

Ichiyama K, Chen T, Wang X, Yan X, Kim BS, Tanaka S, Ndiaye-Lobry D, Deng Y, Zou Y, Zheng P, et al. 2015. The methylcytosine dioxygenase Tet 2 promotes DNA demethylation and activation of cytokine gene expression in T cells. Immunity 42: 613-626.

Ji H, Ehrlich LI, Seita J, Murakami P, Doi A, Lindau P, Lee H, Aryee MJ, Irizarry RA, Kim K, et al. 2010. Comprehensive methylome map of lineage commitment from haematopoietic progenitors. Nature 467: 338-342.

Kaikkonen MU, Spann NJ, Heinz S, Romanoski CE, Allison KA, Stender JD, Chun HB, Tough DF, Prinjha RK, Benner C, et al. 2013. Remodeling of the enhancer landscape during macrophage activation is coupled to enhancer transcription. Mol Cell 51: 310-325.

Klug M, Heinz S, Gebhard C, Schwarzfischer L, Krause SW, Andreesen R, Rehli M. 2010. Active DNA demethylation in human postmitotic cells correlates with activating histone modifications, but not transcription levels. Genome Biol 11: R63.

Klug M, Schmidhofer S, Gebhard C, Andreesen R, Rehli M. 2013. 5Hydroxymethylcytosine is an essential intermediate of active DNA demethylation processes in primary human monocytes. Genome Biol 14: R46.

Kohli RM, Zhang Y. 2013. TET enzymes, TDG and the dynamics of DNA demethylation. Nature 502: 472-479.

Krueger F, Andrews SR. 2011. Bismark: a flexible aligner and methylation caller for Bisulfite-Seq applications. Bioinformatics 27: 1571-1572.

Lam MT, Li W, Rosenfeld MG, Glass CK. 2014. Enhancer RNAs and regulated transcriptional programs. Trends Biochem Sci 39: 170-182.

Langmead B, Salzberg SL. 2012. Fast gapped-read alignment with Bowtie 2. Nat Methods 9: 357-359.

Lister R, Mukamel EA, Nery JR, Urich M, Puddifoot CA, Johnson ND, Lucero J, Huang Y, Dwork AJ, Schultz MD, et al. 2013. Global epigenomic reconfiguration during mammalian brain development. Science 341: 1237905.

Marr AK, MacIsaac JL, Jiang R, Airo AM, Kobor MS, McMaster WR, 2014. Leishmania donovani infection causes distinct epigenetic DNA methylation changes in host macrophages. PLoS Pathog 10: e1004419.

Medzhitov R. 2001. Toll-like receptors and innate immunity. Nat Rev Immunol 1: 135-145.

Monticelli S, Natoli G. 2013. Short-term memory of danger signals and environmental stimuli in immune cells. Nat Immunol 14: 777-784.

Neph S, Vierstra J, Stergachis AB, Reynolds AP, Haugen E, Vernot B, Thurman RE, John S, Sandstrom R, Johnson AK, et al. 2012. An expansive human regulatory lexicon encoded in transcription factor footprints. Nature 489: 83-90.

Ong CT, Corces VG. 2014. CTCF: an architectural protein bridging genome topology and function. Nat Rev Genet 15: 234-246.

Ooi SK, Bestor TH. 2008. The colorful history of active DNA demethylation. Cell 133: 1145-1148.

Ostuni R, Piccolo V, Barozzi I, Polletti S, Termanini A, Bonifacio S, Curina A Prosperini E, Ghisletti S, Natoli G. 2013. Latent enhancers activated by stimulation in differentiated cells. Cell 152: 157-171.

Pastor WA, Aravind L, Rao A. 2013. TETonic shift: biological roles of TET proteins in DNA demethylation and transcription. Nat Rev Mol Cell Biol 14: 341-356.

Pickl WF, Majdic O, Kohl P, Stockl J, Riedl E, Scheinecker C, Bello-Fernandez C, Knapp W. 1996. Molecular and functional characteristics of dendritic cells generated from highly purified CD $14^{+}$peripheral blood monocytes. I Immunol 157: 3850-3859.

Pique-Regi R, Degner JF, Pai AA, Gaffney DJ, Gilad Y, Pritchard JK. 2011. Accurate inference of transcription factor binding from DNA sequence and chromatin accessibility data. Genome Res 21: 447-455.

Qiao Y, Giannopoulou EG, Chan CH, Park SH, Gong S, Chen J, Hu X, Elemento O, Ivashkiv LB. 2013. Synergistic activation of inflammatory cytokine genes by interferon- $\gamma$-induced chromatin remodeling and tolllike receptor signaling. Immunity 39: 454-469.

Quah BJ, Parish CR. 2010. The use of carboxyfluorescein diacetate succinimidyl ester (CFSE) to monitor lymphocyte proliferation. J Vis Exp 44: 2259.

Quintin J, Cheng SC, van der Meer JW, Netea MG. 2014. Innate immune memory: towards a better understanding of host defense mechanisms. Curr Opin Immunol 29: 1-7.

Rada-Iglesias A, Bajpai R, Swigut T, Brugmann SA, Flynn RA, Wysocka J. 2011. A unique chromatin signature uncovers early developmental enhancers in humans. Nature 470: 279-283.

Saeed S, Quintin J, Kerstens HH, Rao NA, Aghajanirefah A, Matarese F, Cheng SC, Ratter J, Berentsen K, van der Ent MA, et al. 2014 Epigenetic programming of monocyte-to-macrophage differentiation and trained innate immunity. Science 345: 1251086.

Santos F, Zakhartchenko V, Stojkovic M, Peters A, Jenuwein T, Wolf E, Reik W, Dean W. 2003. Epigenetic marking correlates with developmental 
potential in cloned bovine preimplantation embryos. Curr Biol 13: $1116-1121$.

Schubeler D. 2015. Function and information content of DNA methylation. Nature 517: 321-326.

Shalek AK, Satija R, Shuga J, Trombetta JJ, Gennert D, Lu D, Chen P, Gertner RS, Gaublomme JT, Yosef N, et al. 2014. Single-cell RNA-seq reveals dynamic paracrine control of cellular variation. Nature 510: 363-369.

Smale ST. 2010. Selective transcription in response to an inflammatory stimulus. Cell 140: 833-844.

Song CX, Szulwach KE, Fu Y, Dai Q, Yi C, Li X, Li Y, Chen CH, Zhang W, Jian $\mathrm{X}$, et al. 2011. Selective chemical labeling reveals the genome-wide distribution of 5-hydroxymethylcytosine. Nat Biotechnol 29: 68-72.

Stadler MB, Murr R, Burger L, Ivanek R, Lienert F, Scholer A, van Nimwegen E, Wirbelauer C, Oakeley EJ, Gaidatzis D, et al. 2011. DNA-binding factors shape the mouse methylome at distal regulatory regions. Nature 480: $490-495$.

Tost J, Gut IG. 2007. DNA methylation analysis by pyrosequencing. Nat Protoc 2: 2265-2275.

Villagra A, Cheng F, Wang HW, Suarez I, Glozak M, Maurin M, Nguyen D, Wright KL, Atadja PW, Bhalla K, et al. 2009. The histone deacetylase
HDAC11 regulates the expression of interleukin 10 and immune tolerance. Nat Immunol 10: 92-100.

Wang D, Garcia-Bassets I, Benner C, Li W, Su X, Zhou Y, Qiu J, Liu W, Kaikkonen MU, Ohgi KA, et al. 2011. Reprogramming transcription by distinct classes of enhancers functionally defined by eRNA. Nature 474: $390-394$.

Yu M, Hon GC, Szulwach KE, Song CX, Zhang L, Kim A, Li X, Dai Q, Shen Y, Park B, et al. 2012. Base-resolution analysis of 5-hydroxymethylcytosine in the mammalian genome. Cell 149: 1368-1380.

Zhou X, Wang T. 2012. Using the Wash U Epigenome Browser to examine genome-wide sequencing data. Curr Protoc Bioinformatics Chapter 10: Unit10.10.

Ziller MJ, Gu H, Muller F, Donaghey J, Tsai LT, Kohlbacher O, De Jager PL, Rosen ED, Bennett DA, Bernstein BE, et al. 2013. Charting a dynamic DNA methylation landscape of the human genome. Nature 500: $477-481$.

Received March 10, 2015; accepted in revised form September 17, 2015. 


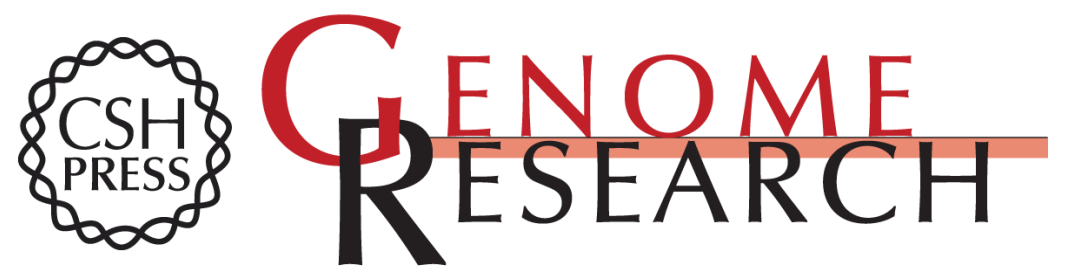

\section{Bacterial infection remodels the DNA methylation landscape of human dendritic cells}

Alain Pacis, Ludovic Tailleux, Alexander M. Morin, et al.

Genome Res. 2015 25: 1801-1811 originally published online September 21, 2015

Access the most recent version at doi:10.1101/gr.192005.115

Supplemental Material

References

Creative

Commons

License

Email Alerting

Service
http://genome.cshlp.org/content/suppl/2015/10/02/gr.192005.115.DC1

This article cites 53 articles, 9 of which can be accessed free at: http://genome.cshlp.org/content/25/12/1801.full.html\#ref-list-1

This article is distributed exclusively by Cold Spring Harbor Laboratory Press for the first six months after the full-issue publication date (see

http://genome.cshlp.org/site/misc/terms.xhtml). After six months, it is available under a Creative Commons License (Attribution-NonCommercial 4.0 International), as described at http://creativecommons.org/licenses/by-nc/4.0/.

Receive free email alerts when new articles cite this article - sign up in the box at the top right corner of the article or click here.

\section{Affordable, Accurate Sequencing.}

To subscribe to Genome Research go to:

https://genome.cshlp.org/subscriptions 